\title{
Utilização de tomografia computadorizada de feixe cônico para identificação de fissuras palatinas: relato de caso clínico
}

\section{Use of cone beam computed tomography for identification of cleft palates: a case report}

\author{
Aline de Freitas Fernandes \\ Cláudia Assunção e Alves Cardoso* \\ Alexandre Camisassa Diniz Leite Greco ${ }^{* * *}$ \\ Gustavo Diniz Greco*** \\ Flávio Ricardo Manzi ${ }^{* * * *}$
}

\section{Resumo}

Objetivo: o objetivo deste trabalho é relatar o caso de uma paciente do sexo feminino, de 14 anos de idade, submetida a alguns exames radiográficos e tomográficos para iniciar um tratamento cirúrgico-reabilitador associado à fissura palatina. Relato de caso: em todos os exames realizados, foi possível identificar tal anomalia, mas, por meio da tomografia computadorizada cone beam e suas reconstruções tridimensionais, foi possível observar toda extensão e profundidade das fendas, contribuindo para realização de um planejamento cirúrgico seguro e previsível. Considerações finais: a tomografia computadorizada de feixe cônico (cone beam) favorece o diagnóstico por imagem em diversas especialidades odontológicas. Porém, devemos saber exatamente quais são as suas indicações e vantagens frente aos exames radiográficos convencionais. Os pacientes com fissuras palatinas necessitam de intervenção cirúrgica de acordo com o tipo e o grau de sua fenda palatina para reabilitação estética e funcional.

Palavras-chave: Fissura palatina. Tomografia computadorizada por raios $X$. Radiologia.

\section{Introdução}

A fissura labiopalatina é uma malformação congênita, que acontece no período embrionário e início do período fetal, em que ocorre a interrupção do processo de formação dos tecidos que formam o palato, e isso gera inúmeras sequelas com as quais o indivíduo terá que conviver por toda sua vida ${ }^{1,2}$. Acredita-se ser de etiologia multifatorial e decorrente de fatores genéticos e ambientais ${ }^{3}$, podendo ser considerados como fatores de risco casos em que a mãe apresenta epilepsia, uso de anti-inflamatórios durante a gestação, poluição, hereditariedade e exposição à radiação ionizante ${ }^{4}$. A incidência média é de 1:700 nascimentos no mundo ${ }^{5}$.

Os portadores de fissura palatina geralmente apresentam dificuldade na alimentação logo após o nascimento ${ }^{6}$. Podem manifestar, também, atraso na aquisição da fala e da linguagem ${ }^{7}$, dificuldades na leitura ${ }^{8}$ e aprendizagem ${ }^{9}$, maloclusões, como mordida cruzada anterior, posterior e oclusão topo a topo ${ }^{10}$, além de apresentar, frequentemente, giro-

Cirurgiã-dentista (Faculdade de Estudos Administrativos - Fead), especialista em Radiologia e Imaginologia Odontológica (Centro de Estudos Odontológicos do IPSEMG - CEO IPSEMG), mestre em Clínicas Odontológicas com ênfase em Radiologia e Imaginologia Odontológica (Pontifícia Universidade Católica de Minas Gerais - PUC Minas), especializanda em Prótese Dentária (Associação Brasileira de Odontologia Seção de Minas Gerais - ABOMG).

Cirurgiã-dentista (PUC Minas), especialista em Radiologia e Imaginologia Odontológica (Faculdade de Odontologia de Piracicaba - Unicamp), mestre em Clínicas Odontológicas (PUC Minas), doutoranda em Odontologia (PUC Minas).

*** Cirurgião-dentista (PUC Minas), especialista em Periodontia (CEO IPSEMG), especialista em Radiologia Odontológica (PUC Minas), mestre em Clínicas Odontológicas com ênfase em Radiologia Odontológica (PUC Minas).

*** Cirurgião-dentista (Faculdade de Odontologia de Itaúna - FOI), especialista em Prótese Dentária (CEO IPSEMG), mestre em Clínicas Odontológicas (PUC Minas), doutor em Odontologia (Universidade Federal de Minas Gerais - UFMG), professor de Prótese Dentária e Clínicas Integradas (Fead).

**** Cirurgião-dentista (Unicamp), mestre em Radiologia Odontológica (Unicamp), doutor em Radiologia Odontológica (Unicamp), professor de Radiologia e Imaginologia Odontológica dos cursos de graduação e pós-graduação da PUC Minas. 
versões, fusões, geminações, microdontias e hipoplasias de esmalte ${ }^{11}$.

Em 1972, foi proposta uma classificação dessas fissuras, tomando como referência o forame incisivo: fissura pré-forame incisivo - são fissuras labiais, unilaterais, bilaterais ou medianas; fissura pós-forame incisivo - são fissuras palatinas, geralmente medianas, que podem localizar-se somente na úvula, no palato, ou envolver todo o palato duro; fissura transforame incisivo - são fissuras que atingem lábio, arcada alveolar e todo o palato, podem ser unilaterais ou bilaterais e são as mais graves ${ }^{12}$.

Também podem ser classificadas como fissuras completas ou incompletas, de acordo com o grau de acometimento das estruturas, e unilaterais, bilaterais ou medianas, de acordo com o lado acomenti$\mathrm{do}^{13}$.

Quando se trata do tratamento cirúrgico, há muitas controvérsias na literatura, já que um fechamento precoce do palato duro possibilita uma fala mais adequada, e o fechamento tardio favorece o crescimento craniofacial ${ }^{14,15}$.

Para fazer um correto diagnóstico e planejamento cirúrgico, podem ser empregados exames radiográficos e tomográficos. Os exames radiográficos são limitados quando se trata de ter acesso a uma estrutura tridimensional precisa, já que ocorre ampliação da imagem e há ausência de um índice anatômico confiável, fato que ocorre porque imagens tridimensionais ficam limitadas a imagens bidimensionais, que apresentam somente altura e largura. Além disso, todo exame radiográfico sofre ampliações pela divergência dos raios X, e essa ampliação depende da distância entre o objeto e o filme e do paralelismo entre eles, ocorrendo distorção da imagem. Esse fato acontece constantemente nas radiografias intrabucais, em casos em que a anatomia não possibilita que o dente e o objeto estejam em uma relação ideal, já que as áreas de interesse estão localizadas em distâncias distintas do filme ${ }^{16}$.

Os exames tomográficos de feixe cônico (cone beam) são mais eficientes do que os radiográficos, já que têm uma projeção ortogonal, na qual os feixes de raios $\mathrm{X}$ estão paralelos e o objeto está perto do sensor ${ }^{15}$. As imagens são feitas em "cortes" da região de interesse, com grande definição, o que permite delimitar áreas de irregularidade em três dimensões ${ }^{17}$.

$\mathrm{Na}$ tomografia computadoriza cone beam, pode-se perceber, também, a relação de profundidade entre as estruturas. Na obtenção da imagem, o feixe de raios $\mathrm{X}$ tem a forma de um cone, que consegue abranger todo o campo de interesse. A cada pulso de radiação, acontece a formação de uma imagem. Após a realização das imagens, elas são unidas, gerando um volume, depois, são novamente fatiadas em cortes axiais, coronais, sagitais, panorâmicos e transversais, por meio de um software específico. As imagens são obtidas em tamanho real, numa escala de 1:1, e, nesse software, podem ser feitas todas as mensurações desejadas, nos diferentes planos ${ }^{16}$. Esse tipo de exame não apresenta sobreposições de estruturas anatômicas ${ }^{18}$ e apresenta uma exposição à radiação relativamente baixa ${ }^{19}$, já que a dose é equivalente à dose recebida em 14 exames periapicais $^{20}$.

Sendo assim, este estudo visa avaliar as vantagens do exame tomográfico cone beam frente às radiografias panorâmicas, periapicais e oclusais, para avaliar em todos os aspectos e qualificar as fissuras palatinas do caso clínico realizado.

\section{Relato de caso}

Paciente de 14 anos de idade, sexo feminino, foi encaminhada a uma clínica radiológica em Belo Horizonte, para realização de exames de imagem radiográficos e tomográficos, a fim de iniciar um tratamento cirúrgico-reabilitador associado à fissura palatina. Durante a preparação para realização dos exames, os responsáveis pela paciente preencheram a anamnese e os devidos protocolos de autorização para uso das imagens em trabalhos acadêmicos e científicos.

Dentre os exames, foram realizados exame radiográfico panorâmico (Figura 1), exames periapicais da região anterior da maxila (Figura 2), exame radiográfico oclusal total de maxila (Figura 3 ) e exame de tomografia computadorizada cone beam de alta resolução, FOV único nas dimensões $5 \mathrm{~mm}$ x 3,75 mm de todo arco superior (Figuras 4, 5 e 6). Tanto o exame panorâmico quanto o exame tomográfico foram obtidos no mesmo aparelho Kodak 9000C 3D. O exame tomográfico foi realizado por meio de aquisição volumétrica em tomógrafo cone beam com $76 \mu \mathrm{m}$ de volume, utilizando os seguintes fatores de exposição: $74 \mathrm{KV}, 10 \mathrm{~mA}, 12$ segundos de exposição.

Em todos os exames por imagem, foi possível avaliar a fissura palatina, mas foi constatado que, no exame tomográfico, conseguiu-se determinar, de maneira mais próxima do real, toda a extensão e a profundidade das fendas, assim como avaliar todos os dentes envolvidos e, a partir de então, auxiliar de forma mais ampla na confecção de um plano de tratamento adequado, para melhor atender na solução do caso da paciente.

A partir dos exames de imagem, a fissura da paciente foi classificada como fissura transforame incisivo bilateral. Nota-se que, no exame tomográfico, pode-se localizar exatamente a região da físsura e mensurar suas dimensões sem nenhuma ampliação ou distorção, como ocorre nos exames radiográficos. Assim, a paciente foi encaminhada ao cirurgião, para posterior planejamento e reabilitação cirúrgica. 


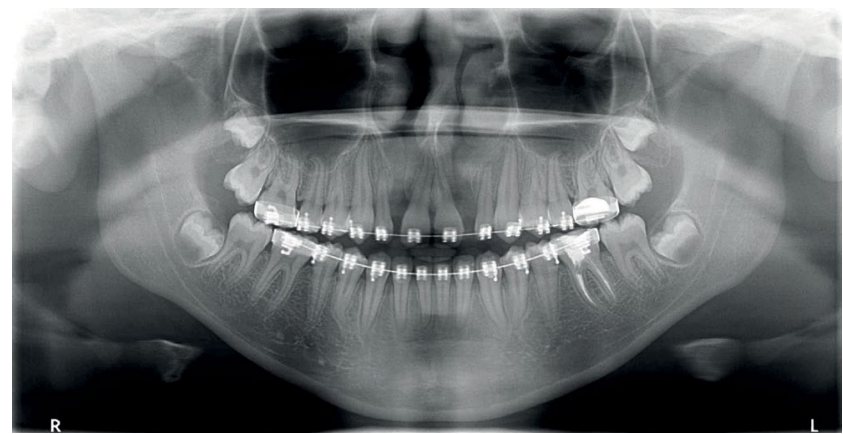

Figura 1 - Exame radiográfico panorâmico

Fonte: dos autores.
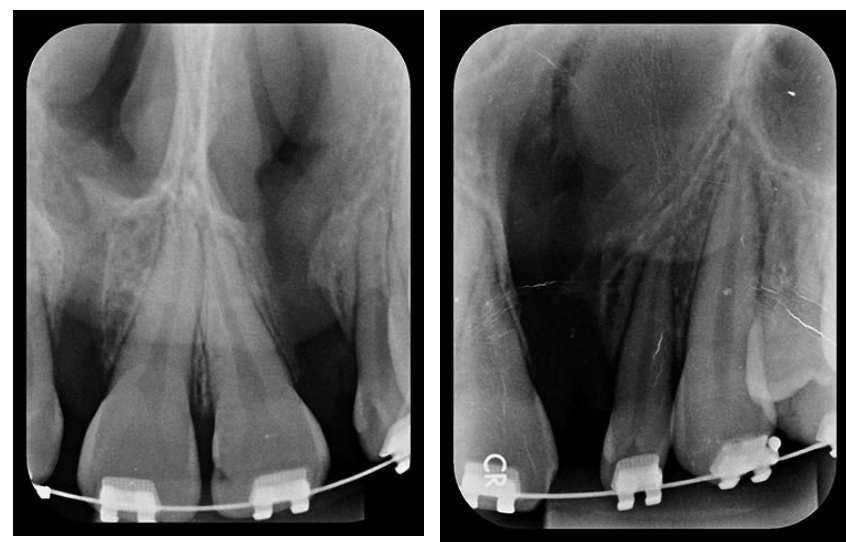

Figura 2 - Exames radiográficos periapicais da região anterior da maFonte: dos autores.

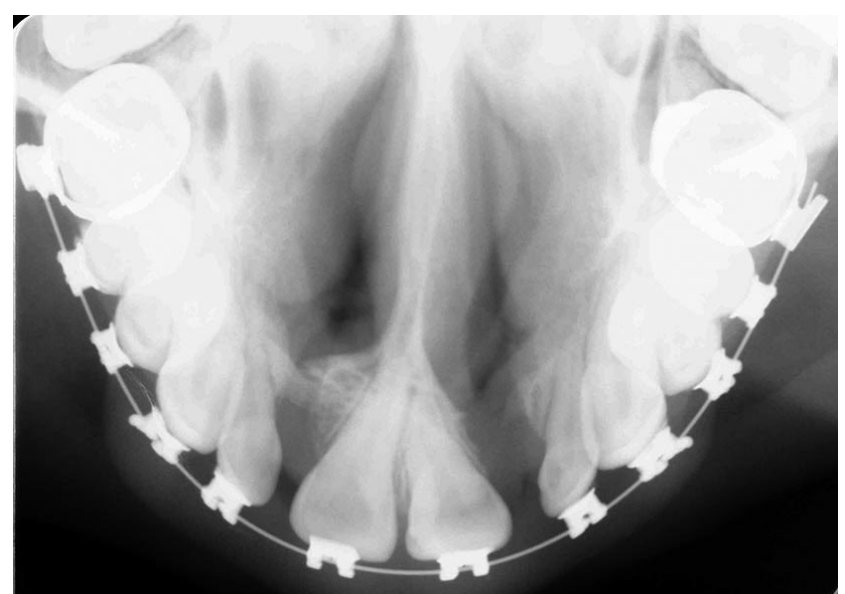

Figura 3 - Exame radiográfico oclusal de maxila total Fonte: dos autores.

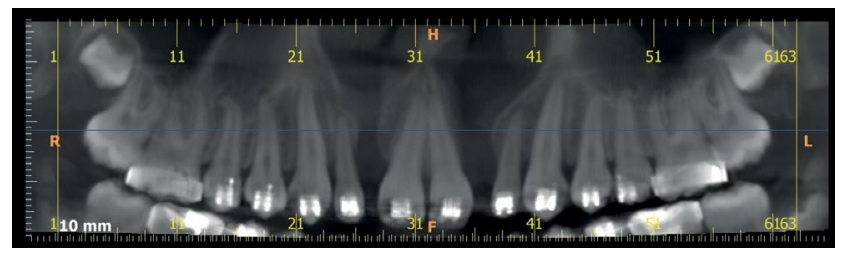

Figura 4 - Reconstrução panorâmica do exame tomográfico Fonte: dos autores.
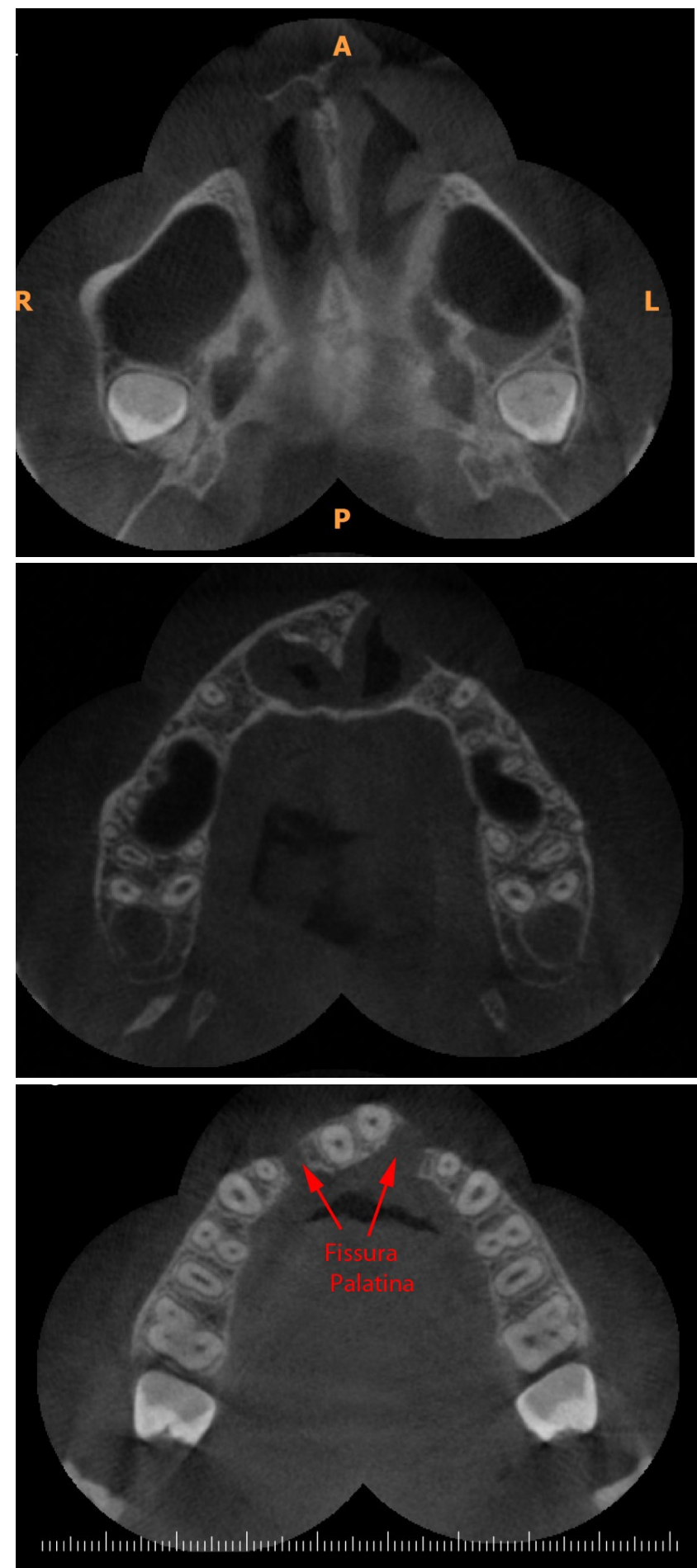

Figura 5 - Cortes axiais do exame tomográfico

Fonte: dos autores. 

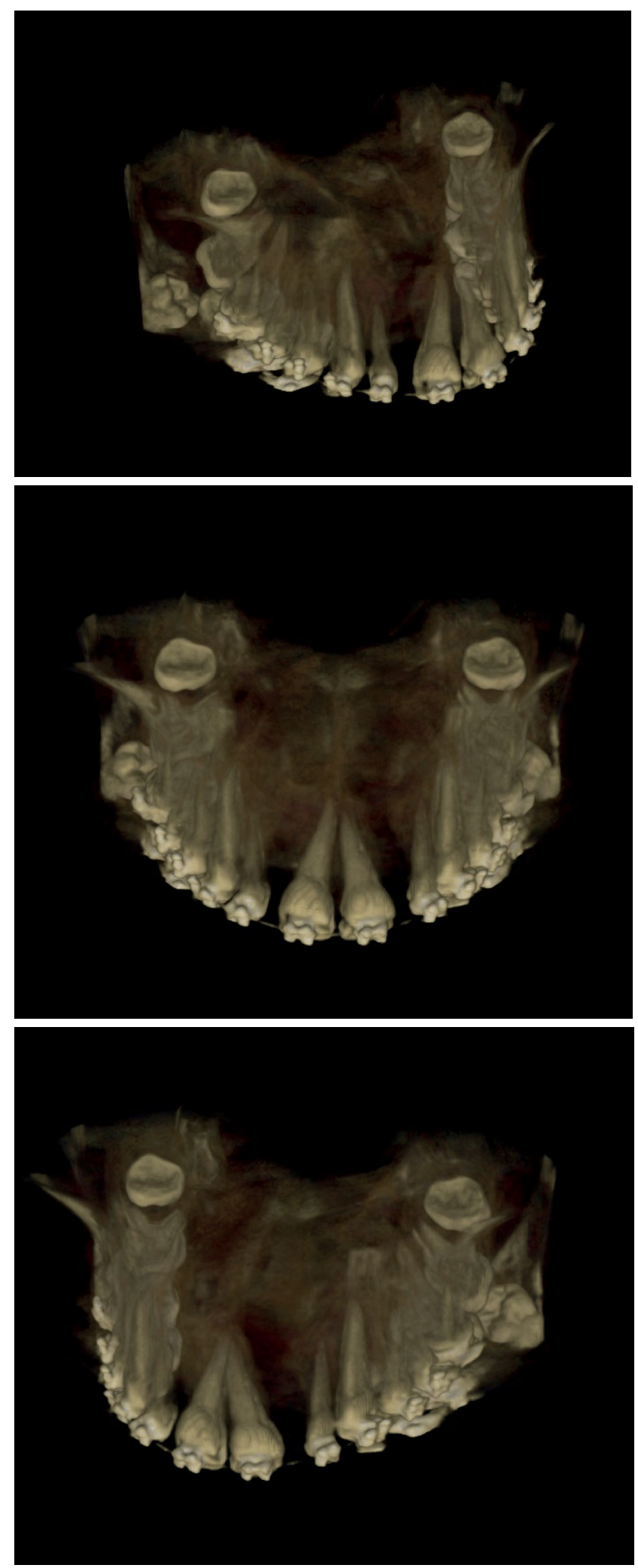

Figura 6 - Reconstrução tridimensional do exame tomográfico

Fonte: dos autores.

\section{Discussão}

As fissuras labiopalatinas ocorrem no período embrionário e início do período fetal, em que há uma interrupção da formação dos tecidos que formam o palato $^{1,2}$. Elas estão entre as malformações congêni- tas mais frequentes que afetam a humanidade, em uma relação que varia entre 1 e 2 indivíduos por 1.000 nascimentos no mundo ${ }^{20}$. Essa prevalência, no Brasil, dependendo da região geográfica, pode ser de 11,89 indivíduos por 10.000 nascimentos e de 3,09 por 10.000 nascimentos ${ }^{21}$.

Diagnósticos com imagens radiográficas, que são imagens bidimensionais, incluem na Odontologia radiografias intrabucais e extrabucais convencionais e panorâmicas, que, apesar de exporem os pacientes a uma baixa dose de radiação, também produzem ampliações, distorções e sobreposições de estruturas anatômicas que podem comprometer a qualidade do tratamento odontológico ${ }^{22^{*}}$

Os exames radiográficos das fissuras geralmente mostram uma ampliação do defeito ósseo alveolar quando comparado ao exame clínico ${ }^{23}$, mas, a princípio, como as radiografias convencionais eram o único método disponível para avaliação de fissuras, o diagnóstico era fundamentado em medidas lineares e avaliações subjetivas de exames oclusais, periapicais e da radiografia panorâmica ${ }^{24}$. Um estudo prospectivo avaliou as diferenças entre imagens radiográficas e tomográficas no diagnóstico e no planejamento do tratamento de caninos superiores impactados. Os resultados mostraram que pode haver diferenças no diagnóstico e no tratamento de caninos superiores impactados, quando são avaliados exames bi e tridimensionais, e que os profissionais confiam mais na exatidão do exame tomográfico ${ }^{22}$.

Em outro estudo, que comparou as radiografias oclusal e periapical com a tomografia computadorizada para avaliar a qualidade da área do enxerto ósseo alveolar, observou-se que as radiografias não tiveram acurácia equivalente à tomografia computadorizada no pós-operatório para a avaliação do sucesso do enxerto ${ }^{25}$.

Para planejamentos individuais, não se deve confiar totalmente em exames bidimensionais, sendo de essencial importância a análise tridimensional, por pontos de referência, linhas e planos ${ }^{26,27}$.

A tomografia computadorizada multislice (TCMS) é um exame preciso quando se trata de avaliação volumétrica das fendas palatinas e alveolares, permitindo realizar uma apreciação válida das dimensões desses defeitos ${ }^{28}$. Porém, de acordo com o projeto Sedentex (2012) da União Europeia de Proteção Radiológica, que determina as condutas referentes ao uso de tomografias computadorizadas, deve-se eleger a tomografia computadorizada de feixe cônico (TCFC) como exame por imagem para avaliação das fissuras palatinas, para que o paciente seja exposto a uma menor dose de radiação. Ainda, de acordo com o mesmo projeto, deve-se optar por um menor volume de exposição, compatível com a área que será avaliada, para que se reduza ainda mais a dose de radiação ${ }^{29}$.

Nesta análise clínica, percebeu-se que, nos exames de tomografia computadorizada de feixe cônico, por meio dos cortes axiais, foi possível avaliar 
a região das fissuras em profundidade, eliminando sobreposições, contornando, assim, uma grande limitação das radiografias periapicais, que é a ausência de profundidade. Sendo assim, para realizar um planejamento cirúrgico mais seguro e previsível, a tomografia computadorizada de feixe cônico é mais indicada.

\section{Considerações finais}

Os exames tomográficos em aparelhos cone beam garantem uma maior exatidão no planejamento de intervenções cirúrgicas, pois permitem a visualização das estruturas em três dimensões, eliminando as deficiências de análises bidimensionais, obtidas pelas radiografias convencionais, contribuindo, assim, para um diagnóstico preciso e para a execução de um planejamento cirúrgico eficaz, que são fundamentais para o sucesso e a previsibilidade do tratamento.

\section{Abstract}

Objective: this study aims to report a case in which the 14 year-old female patient was subjected to a few radiographic and tomographic examinations to initiate a surgical and rehabilitation treatment associated with cleft palate. Case report: all the examinations performed evidenced such anomaly, but the cone beam computed tomography and its three-dimensional reconstructions allowed observing the entire extent and depth of the cracks, contributing to the achievement of a safe and predictable surgical planning. Final considerations: cone beam computed tomography has been favoring diagnostic imaging in several dental specialties. However, we must know exactly what are its indications and advantages over conventional radiographic examinations. Patients with cleft palate need surgical intervention according to the type and degree of their cleft palate for esthetic and functional rehabilitation.

Keywords: Cleft palate. X-ray computed tomography. Radiology.

\section{Referências}

1. Capelloza Filho L, Silva Filho OG. Fissuras lábio-palatais. In: Petrelli E. Ortodontia para Fonoaudiologia. Curitiba: Lovise; 1992. p. 195-239.

2. Ramos MR. A fala no paciente com fissura palatina: uma visão fisiopatológica. In: Carreirão S, Lessa S, Zanini SA. Tratamento das fissuras lábio palatinas. 2. ed. Rio de Janeiro: Revinter; 1996. p. 219-21.

3. Capelozza Filho L, Freitas JAS, Freitas PZ, Capelozza JAZ. Ortodontia na dentadura mista em pacientes com fissura: avaliação da efetividade pelo exame da oclusão na dentadura permanente. Ortodontia 1995; 28(2):71-8.

4. Loffredo LCM, Souza JMP, Yunes J, Souza Freitas JA, Spiri WC. Fissuras lábio-palatais: estudo caso-controle. Rev Saúde Pública 1994; 28:213-7.
5. Akinmoladun VI, Obimakinde OS. Team approach concept in management of orofacial clefts: a survey of Nigerian practitioners. Head Face Med 2009; 5(11):1-5.

6. Redford-Badwal DA, Mabry K, Frassineli JD. Impact of cleft lip and/or palate on nutritional health and oral-motor development. Dent Clin North Am 2003; 47:305-17.

7. Morris H, Ozanne A. Phonetic, phonological and language skills of children with a cleft palate. Cleft Palate Craniofac J 2003; 40:460-70.

8. Richman LC, Eliason MJ, Lindgren SD. Reading disability in children with clefts. Cleft Palate J 1988; 25:21-5.

9. Broder HL, Richman LC, Matheson PB. Learning dis- ability, school achievement and grade retention among children with cleft; a two-center study. Cleft Palate Craniofac J 1998; 35:127-31.

10. Lopes LD, González NZT. Fissuras labiopalatinas. Atuação multidisciplinar precoce. Tratamento ortopédico maxilar e ortodôntico. In: Corrêa MSNP. Odontopediatria na primeira infância. São Paulo: Santos; 2001. p. 627-43.

11. Pini JG, Peres SPBA. Alimentação do lactente portador de lesão lábio-palatal: Aleitamento e introdução alimentar. Rev Nutrição 2001; 14:195-9.

12. Spina V. A proposed modification for the classification of cleft lip and cleft palate. Cleft Palate J 1973; 251-2.

13. Freitas e Silva DS, Mauro LDL, Oliveira LB, Ardenghi TM, Bonecker M. Estudo descritivo de fissuras lábio-palatinas relacionadas a fatores individuais, sistêmicos e sociais. RGO 2008; 56(4):387-91.

14. Nguyen PN, Sullivan PK. Issues and controversies in the management of cleft palate. Clin Plast Surgery 1993; 20(4):671-82.

15. Rohrich RJ, Rowsell AR, Johns DF, Drury MA, Watson DJ. Timing of hard palatal closure: a critical long-term analysis. Plast Reconst Surgery 1996; 98(2):236-46.

16. Oliveira KC, Cruz MA, Alves MB, Maia MMD, Gaião L. Correlação entre dimensões alveolares radiográficas e tomográficas de pacientes com fissuras palatinas. Pesq Bras Odontoped Clin Integrad 2012; 12(1):19-25.

17. Garib DG, Raymundo Jr R, Raymundo MV, Raymundo DV, Ferreira SN. Tomografia computadorizada de feixe cônico (cone beam): entendendo este novo método de diagnóstico por imagem com promissora aplicabilidade na ortodontia. Rev Dent Press Ortodon Ortop Facial 2007; 12(2):139-56.

18. Rosenstein SW, Long Jr RE, Dado DV, Vinson B, Alder ME. Comparison of 2-D calculations from periapical and occlusal radiographs versus 3-D calculations from CAT scans in determining bone support for cleft-adjacent teeth following early alveolar bone grafts. Cleft Palate Craniofac J 1997; 34(3):199-205.

19. Schneiderman ED, Xu H, Salyer KE, Faap F. Characterization of the maxillary complex in unilateral cleft lip and palate using cone-beam computed tomography: a preliminary study. J Craniofac Surg 2009; 20(2):1699-710.

20. Parks ET. Computed tomography applications for the dentistry. Dent Clin North Am 2000; 44(2):371-94.

21. Petrelli E. Ortodontia para Fonoaudiologia. Curitiba: Lovise; 1992.

22. Haney E, Gansky SA, Lee JS, Johnson E, Maki K, Miller AJ, et al. Comparative analysis of traditional radiographs and cone-beam computed tomography volumetric images in the diagnosis and treatment planning of maxillary impacted canines. Am J Orthod Dentofacial Orthop 2008; 137(5):590-7.

23. Trindade IEK, Silva Filho OG. Fissuras labiopalatinas: uma abordagem interdisciplinar. São Paulo: Santos; 2007. 
24. Aurouze C, Moller KT, Bevis RR, Rehm K, Rudney J. The presurgical status of the alveolar cleft and success of secondary bone grafting. Cleft Palate Craniofac J 2000; 37(2):17984.

25. 25. Nightingale C, Whiterow H, Reid FDA, Edler R. Comparative reproducibility of three methods of radiographic assessment of alveolar bone graft. Eur J Orthod 2003; 25(1):35-41.

26. 26. Wörtche R, Hassfeld S, Lux CJ, Müsig E, Hensley FW, Krempien R, et al. Clinical application of cone beam digital volume tomography in children with cleft lip and palate. Dentomaxillofac Radiol 2006; 35(2):88-94.

27. 27. Kim KR, Kim S, Baek SH. Change in grafted secondary alveolar bonein patients with UCLP and UCLA: a three- dimensional computed tomography study. Angle Orthod 2008; 78(4):631-40.

28. 28. Choi HS, Choi HG, Kim SH, Park HJ, Shin DH, Jo DI, et al. Influence of the alveolar cleft type on preoperative estimation using 3D CT assessment for alveolar cleft. Arch Plast Surg 2012; 39(5):477-82.

29. 29. SEDENTEXCT guidelines. Safety and Efficacy of a New and Emerging Dental X-ray Modality. Radiation protection no. 172: cone beam CT for dental and maxillofacial radiology (evidence-based guidelines). 2012. Disponível em URL: http://www.sedentexct.eu/files/radiation_protection_172.pdf.

\section{Endereço para correspondência:}

Aline de Freitas Fernandes

Rua Professor Miguel de Souza, 359, apto 502, Buritis

CEP 30575-255 Belo Horizonte, MG, Brasil

Telefone: 55 (31) 98399-2898.

E-mail: draalinefernandes@hotmail.com

Recebido: 16/05/2016. Aceito: 27/07/2017. 